\title{
A NOVEL PRECURSOR IN PREPARATION AND CHARACTERIZATION OF NICKEL OXIDE (NIO) AND COBALT OXIDE (CO3O4) NANOPARTICLES (NPS)VIA AQUEOUS CHEMICAL GROWTH (ACG) TECHNIQUES
}

\author{
A.R Junejo ${ }^{1}$, S.Memon ${ }^{1}$, S.Pathan ${ }^{2}$ \\ ${ }^{1}$ Institute of Information and Communication Technology, University of Sindh Jamshoro, \\ ${ }^{2}$ Center of Excellence and Analytical chemistry, University of Sindh Jamshoro
}

\begin{abstract}
Synthesis of Nickel Oxide ( $\mathrm{NiO}$ ) nanoparticles and cobalt oxide (CO3O4) materials synthesis by aqueous chemical growth (ACG) Techniques. Oxide based material having a wide band gap, and suitable for optical devices, Optoelectronic devices, UV photodetector, and Light emitting diode LEDs. The analysis and characterizationof Nickel Oxide (NiO) and cobalt oxide (CO3O4) nanoparticles by(1) X-ray diffraction (XRD), (2) Scanning electron microscopy (SEM), and (3) Ultraviolet-visible (UV-Vis) spectroscopy.
\end{abstract}

\section{Key words}

Nickel Oxide (NiO), Cobalt oxide (CO3O4), an oxide materials, Nanostructures.

\section{INTRODUCTION}

Challenging to find new Nano material focus on Metal oxide due to their special characteristics. Due to accomplished CMOS process and unique properties of the material, Silicon has dominated the semiconductor device industry since 1960s [2]. However less mobility and low band-gap (lower breakdown voltage) of silicon limits the current carrying and output power generation capability of traditional silicon based devices. Therefore compound semiconductors have become the dominant materials for the applications like Light Emitting Diodes (LEDs), Laser Diodes (LDs) and Radio Frequency (RF) devices. In this standpoint the typical III-V compound semiconductors successfully get their place in semiconductor device industry especially in the field of optoelectronic due to their significant properties such as wide band gap, High electron mobility and wide spectral range.Metal oxides are dynamically used in the semiconductor industry, such as most of the chips used in computers contain an oxide component. The synthesis, characterization and applications of the metal oxide nanomaterials become challenging day by day among researchers because of many unique features associated with nanometer size of the particles [3-5].Among many wide band gap semiconductors such as $\mathrm{NiO}, \mathrm{ZnO}, \mathrm{SiC}, \mathrm{GaN}$, etc., $\mathrm{ZnO}$ as n-type material came under the scientific spotlight due to its distinguished execution in fields like electronics, optics and photonics. Among various p-type materials, $\mathrm{NiO}$ is a promising material having a wide band gap $(3.6 \mathrm{eV}$ to $4.0 \mathrm{eV})$ which is suitable for optical devices. $\mathrm{NiO}$ has also been studied widely for applications in transparent conductive films, electrochromic devices and heterogeneous catalysts along with lithium-ion batteriesand chemical sensors [6-9]. and high adsorption capability pave the way to make desirable surroundings for the immobilization of biomolecule and bettered bio-sensing features [10-13]. Therefore it is of great importance to select that metal oxide nanostructure, which favor's most the immobilization of the biomolecules. Beside this they are very economical, less toxic and have great flexibility in structures/morphology. Among the reported transition metal oxides, the cobalt oxide $\left(\mathrm{Co}_{3} \mathrm{O}_{4}\right)$ has shown a lot of promise.

In the present work $\mathrm{NiO}$ and $\mathrm{Co}_{3} \mathrm{O}_{4}$ and their composite nanostructures have been synthesized and used for device fabrication in different fields. And the main Objective Growth of $\mathrm{NiO}$ nanostructures $\mathrm{NiO}$ has been used as p-type material due to its potential role in luminescence applications along with it's In this study $\mathrm{NiO}$ nanostructures have been developed. Cobalt oxidenanostructures are synthesized by hydrothermal technique, which are investigated for their luminescence properties. 


\section{RELATED WORK}

The synthesis, characterization and applications of the metal oxide nanomaterials become challenging day by day among researchers because of many unique features associated with nanometer size of the particles [3,4]. Generally the size of the particle has influence on the properties in two aspects.

i. $\quad$ Change in structural characteristics, such as the lattice symmetry and cell parameters.

ii. Due to the oxygen vacancies in oxide nanoparticles atomic arrangement of the materials varies from that in the bulk, along with occupation of electronic states above the valance band. In addition to this an enhancement in the chemical activity of the system also arises.

These properties of nanostructured oxides opened a door for applications Moreover, these materials can be further functionalized by surface and structure modification. The good thermal and chemical stability of these inorganic materials enable them to be widely used.Properties of Nickle Oxide $(\mathrm{NiO})$. Some important application of nickel oxide in large scale optical switching glazing, anti-ferromagnetic material, electrochromic display devices, lithium ion batteries (battery cathode) and functional layer material for gas chemical sensors, catalysis andelectro chromic films a[1-5].

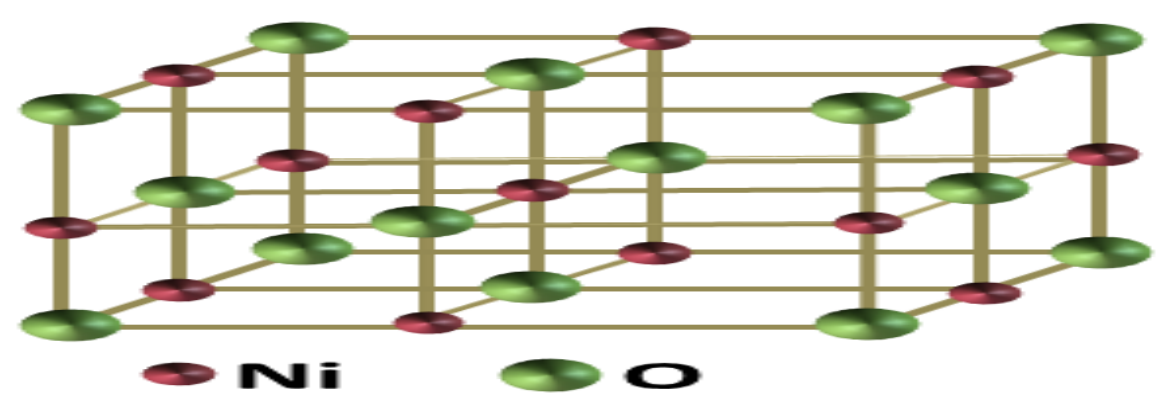

Figure 1.Shows the face centered cubic (fcc) crystal structure of NiO. (Reproduced)

Semiconducting Propertiesof $\mathrm{NiO}$ thin film is nearly transparent, wide band-gap semiconductor, and nonstoichiometric which shows p-type semiconductor conducting behavior $[1,2,9]$. NiO (Nickelous oxide) nanoparticles is an important metal oxide with a wide band gap of about 3.8 $\mathrm{eV}$, acts as a P-type semiconductor and can be used as an emitter of $341 \mathrm{~nm}$, Properties of Cobalt Oxide $\left(\mathrm{Co}_{3} \mathrm{O}_{4}\right)$ semiconducting, capacitive characteristics and environmental friendliness. There are two types of stable oxide of cobalt known as $(\mathrm{CoO})$ and $\mathrm{Co}_{3} \mathrm{O}_{4}$, both of them are stable at room temperature. Cobalt (II, III) oxides are inorganic and mixed valence compound[15].

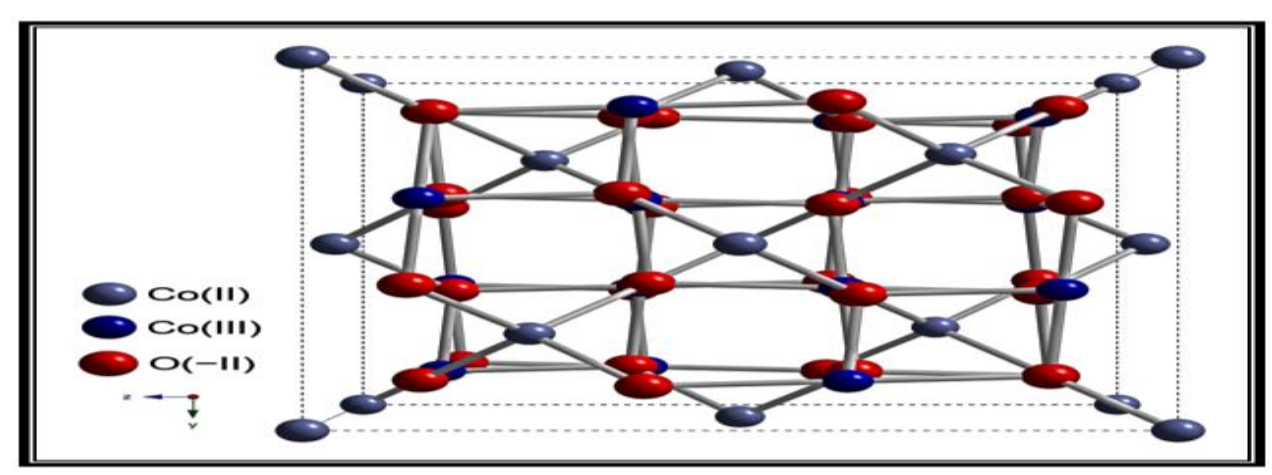

Figure 2. The unit cell structure of $\mathrm{Co}_{3} \mathrm{O}_{4}$.(Reproduced)

$\mathrm{Co}_{3} \mathrm{O}_{4}$ is magnetic p-type semiconductor material with direct band gaps of 1.48 and $2.19 \mathrm{ev}$, however $1.6 \mathrm{ev}$ is also reported in literature. $\mathrm{Co}_{3} \mathrm{O}_{4}$ is extensively used as catalyst, solid state sensors, in pigment, electrochromic devices, solar energy absorber magnet and capacitor [15, 16].Cobalt oxides get much attention in past few years due to its capacitive characteristics In present study we are interested to investigate the Luminescence properties of cobalt oxide to use it as low power LEDs. 


\section{SYNTHESIS TECHNIQUES}

Synthesis is the back bone for the growth of nanostructures. In order to discover novel physical properties and to realize possible applications of nanostructures and nanomaterials, the first corner stone in nanotechnology is to fabricate and process nanomaterials and nanostructures at their particular scale to achieve the best results. Mainly there are two approaches used for the synthesis of nanomaterials according to the growth process.

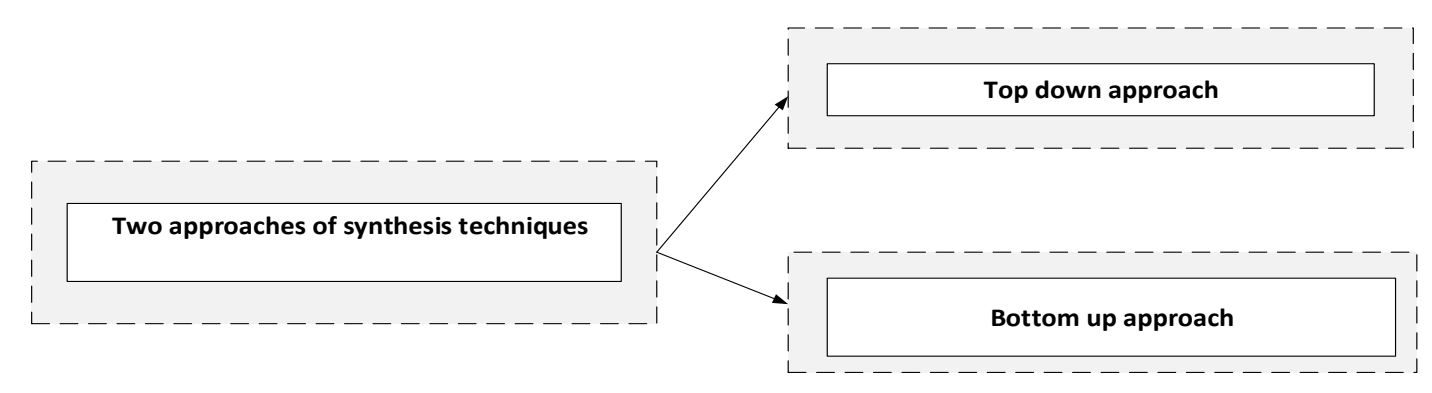

Fig.no. 3. Basic synthesis techniques

\subsection{Hydrothermal Synthesis.}

Hydrothermal Synthesis orAqueous chemical growth (ACG) is a common and simple, low temperature growth technique. Hydrothermal synthesis involves crystallizing materials from high temperature aqueous solutions under autogenous pressure. Hydrothermal synthesis was typically carried out in a steel pressure vessel known as an autoclave. Within the autoclave a temperature gradient is established and maintained at the opposite ends of the growth chamber such that the hotter end aids dissolution and the cooler end aids crystal growth[7, 3].

A precursor solution is required for the growth of $\mathrm{NiO}$ nanostructures as described earlier in this method. Seeded substrates were attached to a Teflon sample holder and dipped into the precursor solution for incubation at a temperature of $90-95^{\circ} \mathrm{C}$ for $5-6$ hours. The ACG method was used for the growth of $\mathrm{NiO}$ nanostructure. A piece of substrate around $1 \mathrm{~cm} \times 2 \mathrm{~cm}$ in size was cleaned by the standard cleaning procedure as described earlier. Then $\mathrm{NiO}$ nanoparticles (seeds) solution was deposited on the substrate by using spin coater at a speed of $4000 \mathrm{rpm}$. This process was repeated three times to achieve homogeneous and dense seed layer on the surface of the substrate and then it was heated for several minutes for getting good adhesion of nano particles on the surface of substrate. Now the sample was attached to the sample holder and immersed into the precursor's solution, which was prepared by dissolving NiN and HMT in $200 \mathrm{ml}$ DI water. This container was placed into the oven held at a constant temperature of $90{ }^{\circ} \mathrm{C}$ for 6 hours. After the growth the sample was washed by DI water and dried by nitrogen. After the growth of $\mathrm{NiO}$ nanostructure the sample was ready to be used for preparing low power LEDs. Therefore structural analysis is essential, so SEM, XRD, C.L and UV techniques were used for the characterization of the prepared sample. The same steps are followed for the growth of $\mathrm{Co}_{3} \mathrm{O}_{4}$ (cobalt oxide) nanostructure. Hydrothermal technique has large number of advantages over the other synthesis techniques. It is very easy to handle, consume low power, required small time for the growth of nanostructure and cheap method.

\section{Characterization and Analysis.}

Therefore structural analysis is essential, so SEM, XRD, C.L and UV techniques were used for the characterization of the prepared sample. The same steps are followed for the growth of $\mathrm{Co} 3 \mathrm{O} 4$ (cobalt oxide) nanostructure.X-ray diffraction (XRD) is one of the key methods available to determine atomic structure, including crystallite size, phase, and phase composition. In order to investigate the surface morphology of the grown $\mathrm{NiO}$ and $\mathrm{Co}_{3} \mathrm{O}_{4}$ nanostructures scanning electron microscope technique was employed. Figure 6 is revealing the low and high magnification SEM images of the morphology of $\mathrm{NiO}$ and $\mathrm{Co}_{3} \mathrm{O}_{4}$ nanostructures grownon glass substrate. It is clear from Figure 6.1 (a) that $\mathrm{NiO}$ nanostructures are looks like honey comb. Beside this they are very dense and well uniform as shown in figure 6.1 (a)\& (b). In addition to this 6.1 (c) \& (d) are showing the morphology of as grown $\mathrm{Co}_{3} \mathrm{O}_{4}$ nanostructures. The grass like nanostructures of Co3O4are uniformly distributed all over the substrate and they are interconnected as well. 


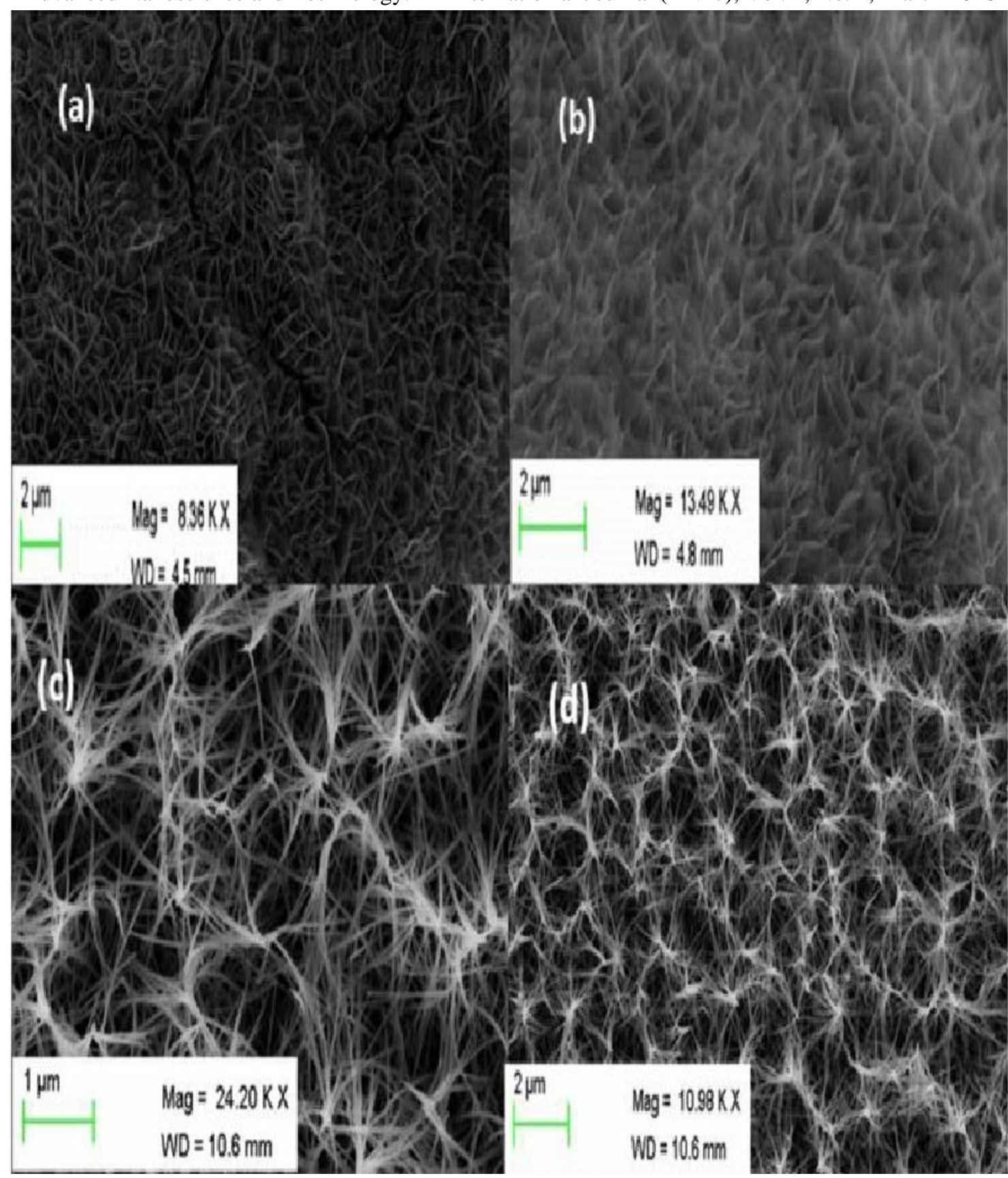

Fig.no. 4: SEM image for $\mathrm{NiO}$ nanostructures (a) \& (b) and Cobalt Oxide nanostructure (c) \& (d)

In order to examine the crystal quality of grown $\mathrm{NiO}$ and $\mathrm{Co}_{3} \mathrm{O}_{4}$ nanostructures XRD measurements have been performed.The XRD scan $\left(0.1^{\circ} / \mathrm{sec}\right)$ was performed in the $2 \Theta$ range of $25^{\circ}-70^{\circ}$ for $\mathrm{NiO}$ and $15^{\circ}-70^{\circ}$ for $\mathrm{Co}_{3} \mathrm{O}_{4}$ nanostructures. Figure $6.2(\mathrm{a})$ is conforming that $\mathrm{NiO}$ nanostructures are cubic in crystallinity and the presence of rings in nanostructures showing the nanoflakes nature of $\mathrm{NiO}$. The appearance of $\mathrm{Ni}$ (111) reflectionis confirming the presence of nanocrystalline fcc $\mathrm{Ni}$ grains in the prepared sample.The highest appeared peak at (111) and the peak at (200) of $\mathrm{NiO}$ are clearly visible in obtained spectra. The XRD study exhibited the facecentered cubic phase crystalline structure of NiO.The XRD scan showed in Figure 6.2(b)is revealing the pure phase of $\mathrm{Co}_{3} \mathrm{O}_{4}$ nanostructures. The XRDspectrum in figure 6.2 (b)is showingmany peaks related to $\mathrm{Co}_{3} \mathrm{O}_{4}$ nanostructures. These peaks are in well agreement with JCPDS card no. 05-0661). A relatively weak peak related to a monoclinic phase of $\mathrm{Co}_{3} \mathrm{O}_{4}$ appears at 38.7 and it corresponds to the (111) plane. The peaks identified as $(112)$ and $(1,1,-3)$ are reflections for $\mathrm{Co}_{3} \mathrm{O}_{4}$. This study has shown that the fabricated nano structure is only composed of $\mathrm{Co}_{3} \mathrm{O}_{4}$ materials and the obtained results are consistent with the SEM analysis. 


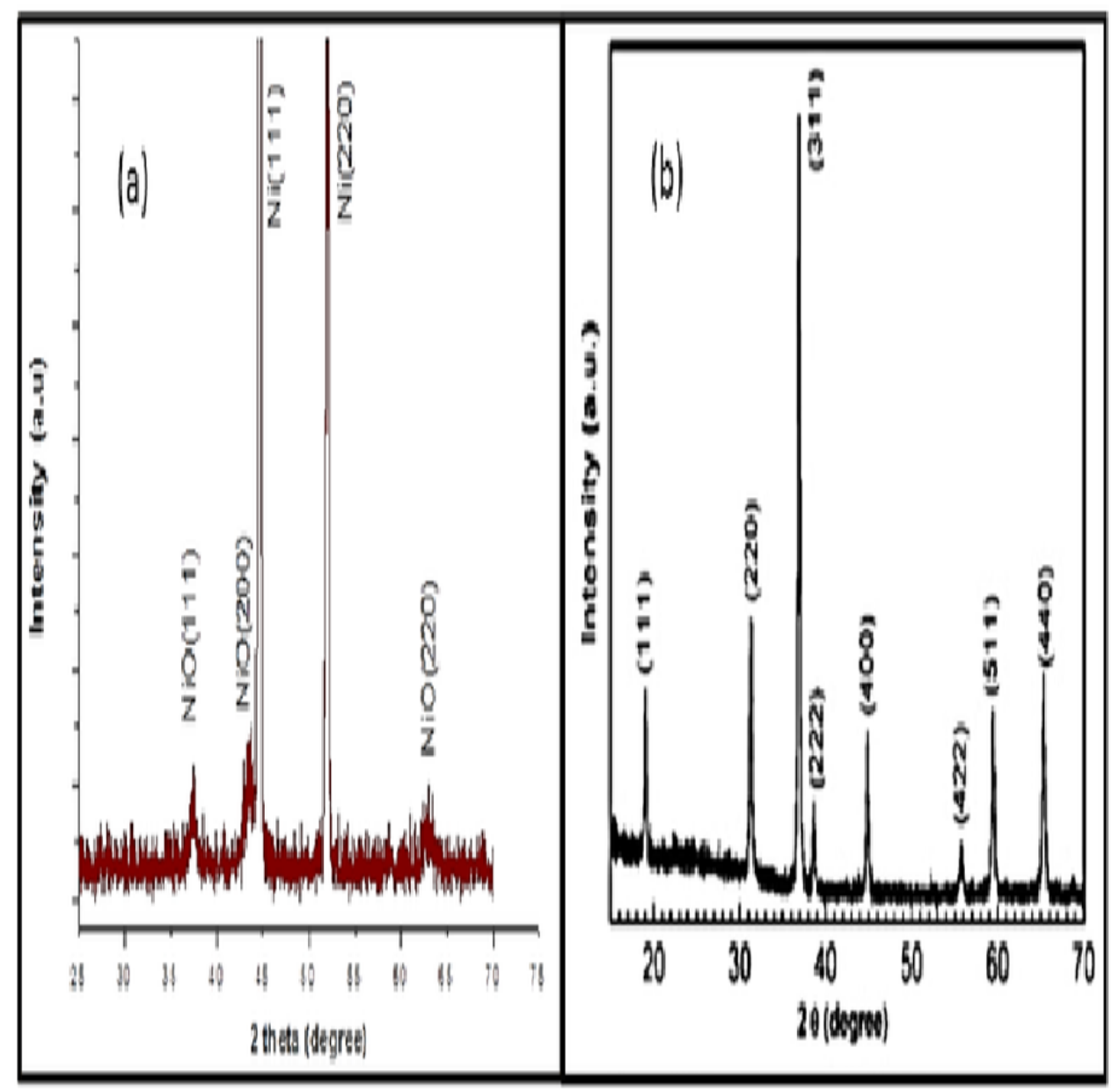

Fig.no 5. X Ray diffraction pattern of $\mathrm{NiO}(\mathrm{a})$ and $\mathrm{Co}_{3} \mathrm{O}_{4}(\mathrm{~b})$ nanostructure

For the study of luminescence nature of grown $\mathrm{NiO}$ and $\mathrm{Co}_{3} \mathrm{O}_{4}$ nanostructures, UV-vis spectroscopywas performed at room temperature. Figure 6.3(a) has shown enhanced UV luminescence at room temperature for $\mathrm{NiO}$ nanostructures while $\mathrm{Co}_{3} \mathrm{O}_{4}$ showed very weak luminesce in the visible region as shown in figure 6.3(b). We have measured the pure $\mathrm{Co}_{3} \mathrm{O}_{4}$ nanostructuresand we have not detected any strong luminesce in the visible region.

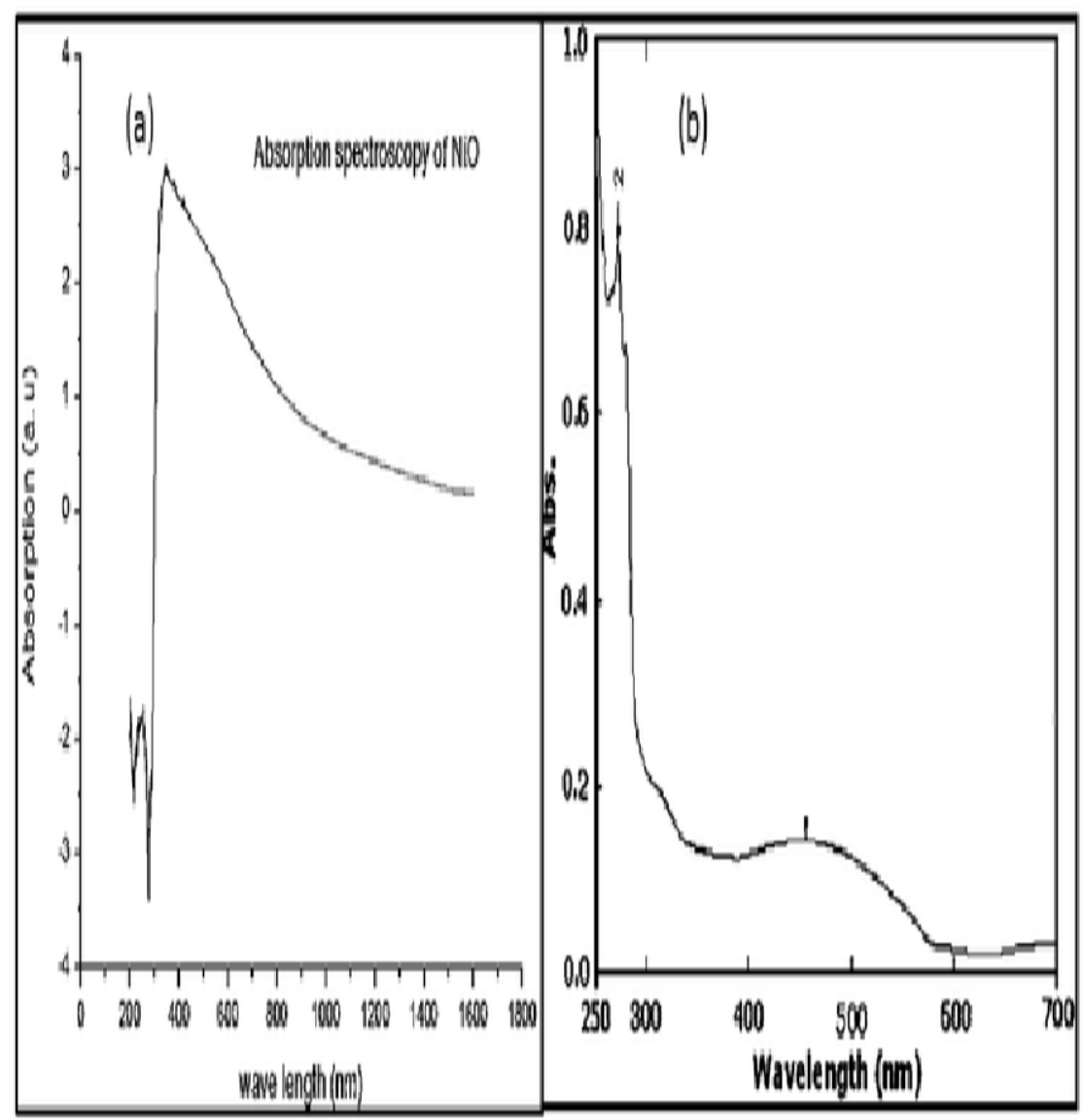

Fig.no.6 (a) UV -vis spectrum of $\mathrm{NiO}$ and (b) $\mathrm{UV}$-vis spectrum of $\mathrm{Co}_{3} \mathrm{O}$. 
Cathodoluminescentstudy was done in order to confirm luminescein the as grown nanostructures of $\mathrm{NiO}$ and $\mathrm{Co}_{3} \mathrm{O}_{4}$. CL spectra are practically independent on the electron beam energy, only a marginal increase of the relative intensity of the visible emission has been detected. It is clear from the graph shown by the figure 6.4 that $\mathrm{NiO}$ has an intense peak about $500 \mathrm{~nm}$ with intensity of around 1400 a.u.This result proves that $\mathrm{NiO}$ nanostructures are good to be used for LEDs.

We have measured the pure $\mathrm{Co}_{3} \mathrm{O}_{4}$ nanostructures and we have not detected any luminescence in $\mathrm{UV}$ or in the visible region therefore cobalt oxide nanostructure are not suitable for LEDs.

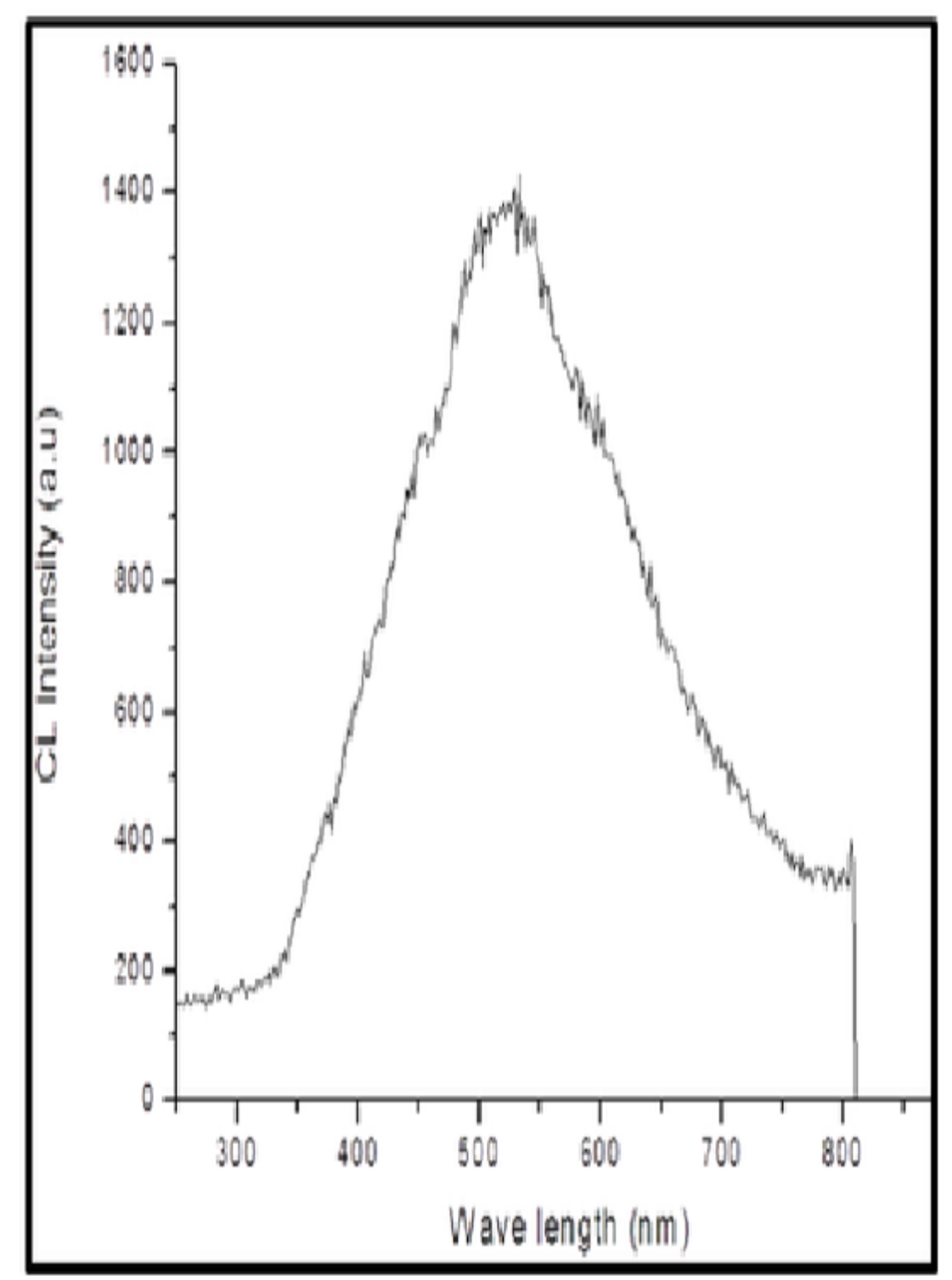

Fig.no 7 : CL spectra of $\mathrm{NiO}$

\section{CONCLUSION}

In this study, $\mathrm{NiO}$ and $\mathrm{Co}_{3} \mathrm{O}_{4}$ nanostructures were fabricated on the glass substrate by employing aqueous chemical growth method. The structural study of grown nanostructures of $\mathrm{NiO}$ and $\mathrm{Co}_{3} \mathrm{O}_{4}$ showed uniform distribution, high density and good crystal quality. Moreover, the $\mathrm{NiO}$ nanostructuresshowed good UV-visible absorption behavior. On the other hand $\mathrm{Co}_{3} \mathrm{O}_{4}$ nanostructures have not showed strong UV-visible absorption behavior suitable for LEDs. CL investigation of $\mathrm{NiO}$ nanostructuresshowed the excellent luminescence, but $\mathrm{Co}_{3} \mathrm{O}_{4}$ nanostructures did not showed any luminescence in that investigation. This also confirmed the fruitfulness of $\mathrm{NiO}$ and unfruitfulness of $\mathrm{Co}_{3} \mathrm{O}_{4}$ in the fabrication ofLEDs.

\section{Reference}

[1] Marcos Fernández-Garcia, José A. Rodriguez, Metal Oxide Nanoparticles, Brookhaven National Laboratory 2007.

[2] D.J. Frank, R.H. Dennard, E. Nowak, P.M. Solomon, et al., Proceedings of the IEEE89 (2001).

[3] J.P. Jolivet, Metal Oxide Chemistry and Synthesis: From Solution to Solid State, Wiley, Chichester (2000). 
Advanced Nanoscience and Technology: An Internation al Journal (ANTJ), Vol. 2, No. 1, March 2016

[4] G. R. Patzke, F. Krumeich, R. Nesper, Angew. Chem. Int. Ed. 41 2446-2461 (2002).

[5] Y. Y. Wu, H. Q. Yan, M. Huang, B. Messer, et al., Chem. Eur. J. 81261 (2002).

[6] S. Strite, H. Morkoc, J. Vac. Sci. Technol. B10 1237 (1992).

[7] Mushtaque Hussain, synthesis, characterization and applications of oxide nanostructures, 12-13 (2014)

[8] Guoxiu Wang, Xiaoping Shen, Josip Horvat, Bei Wang, Hao Liu, David Wexler, and Jane Yao, Hydrothermal Synthesis and Optical, Magnetic, and Supercapacitance Properties of Nanoporous Cobalt Oxide Nanorods (2009)

[9] Venter A, Botha JR. Optical and electrical properties of $\mathrm{NiO}$ for possible dielectric applications. S Afr J Sci. 2011;107(1/2), Art. \#268, 6 pages. DOI: 10.4102/sajs. v107i1/2.268

[10] P. RSabah Ibrahim Abbas, Ahmed Qasim Ubaid. Structural, Optical and photoluminescence Properties of Nanocrystalline NiO Thin Films. Journal of advances in Physics, Vol .6(1)

[11] Z. Y. Wu, $, \dagger, \ddagger$ C. M. Liu, $\S$ L. Guo, ${ }^{*}, \S$ R. Hu, $\uparrow$ M. I. Abbas, $\uparrow$ T. D. Hu, $\uparrow$ and H. B. Xu§. Structural Characterization of Nickel Oxide Nanowires by X-ray Absorption Near-Edge Structure Spectroscopy. J. Phys. Chem. B 2005, 109, 2512-2515

[12] Huang, M. H.; Wu, Y.; Feick, H.; Tran, N.; Weber, E.; Yang, P. AdV. Mater. 2001, 13, 113. 